\title{
COMUNIDAD, REALIDAD Y PRAGMATISMO
}

Alberto M. Damiani*

\section{Resumen}

El propósito de este trabajo es explicar la concepción de la realidad sostenida por Charles Sanders Peirce. El trabajo comienza con una presentación de dicha concepción en tres artículos de Peirce: : "The fixation of bclief” (1877) y "How to make our ideas clear" (1878) and "Questions concerning certain facultics claimed for man" (1868). Luego se examinan algunas críticas a dicha concepción. La conclusión es que esas críticas dependen de una comprensión incorrecta de esta concepción.

Palabras clave: Peirce, comunidad, realidad, pragmatismo, pragmática.

\section{Abstract}

The aim of this paper is to explain Charles Sanders Peirce' conception of reality. The paper begins with a presentation of this conception in three works of Peirce: "The fixation of belief" (1877) y "How to make our ideas clear" (1878) and "Questions concerning certain faculties claimed for man" (1868). After that, some critics to this conception are examined. The conclusion is that these critics depend of a misunderstanding of this conception.

Keywords: Peirce, community, reality, pragmatism, pragmatics.

Los problemas teóricos y prácticos plantean a los seres humanos tareas que sólo pueden solucionarse colectivamente. La búsqueda de la mejor solución para cualquier problema no es nunca la experiencia de una conciencia individual. Esta búsqueda implica la necesidad de un diálogo argumentativo (al menos virtual) entre los interesados por la solución del problema en cuestión. Para participar de ese diálogo se requieren ciertas condiciones. En primer lugar, quien pretenda tener la última palabra sobre la solución del problema no parece ser un buen participante de un diálogo argumentativo. Él cree estar en la situación de quien ya no necesita aprender

\footnotetext{
* Investigador del CONICET. Profesor Titular de Historia de la Fílosofía Moderna cn la Universidad Nacional de Rosario. Dirccción electrónica: damial@,filo.uba.ar
} 
nada de las opiniones ajenas ni de los posibles exámenes críticos de su propuesta para solucionar el problema en cuestión. Por ello, la pretensión de tener ya la última palabra sobre un problema es un obstáculo para la búsqueda argumentativa de su solución. Sin embargo, es importante resaltar otra condición que deben cumplir quienes se proponen solucionar argumentativamente un problema. Éstos no deben ocuparse superficialmente del problema, como si se tratara (sólo) de una conversación entretenida, sino buscar la opinión que puede valer como la solución del problema. Por ello, al parecer, los participantes de un diálogo argumentativo deben necesariamente buscar una "opinión última".

En el presente trabajo, quisiera presentar esquemáticamente el uso que Charles Sanders Peirce hizo de la noción de "opinión última" para aclarar el significado de los términos "realidad" y "verdad". En este uso puede encontrarse una clave para disolver muchas de las perplejidades propias tanto de otros representantes del pragmatismo decimonónico, como de algunos neo-pragmatistas contemporáneos. Comenzaré presentando algunos argumentos sostenidos por Peirce en dos artículos: "The fixation of belief" (1877) y "How to make our ideas clear" (1878) ${ }^{1}$ (1). Luego distinguiré dos aspectos en el tratamiento del problema de la cognoscibilidad de lo real, a saber, un aspecto que puede denominarse "crítico semiótico" o "crítico de sentido", referente a las condiciones de sentido del uso de los signos, y un aspecto epistemológico, referente a la capacidad humana para conocer la realidad (2). Este segundo aspecto puede ser aclarado, al menos en parte, recurriendo a otro trabajo de Peirce, titulado "Questions concerning certain faculties claimed for man" (1868) (3). La cuestión sexta de este trabajo de Peirce ha sido objeto de un reciente análisis crítico de Daniel Kalpokas, quien pretende refutar a Peirce y sostener la siguiente tesis: no podemos saber si hay incognoscibles ${ }^{2}$. Frente a estas pretensiones,

1 Cfr. Charles Sanders Peirce, Pragmatism and Pragmaticism, en: Collected Papers of Charles Sanders Peirce, vol. V., Cambridge, Cambridge University Press, 1934. En adelante C. P.: 5. seguido de los números de parágrafos. Cito la traducción de José Vericat: Charles Sanders Peirce, Un bombre, un signo. El pragmatismo de Peirce, Barcelona, Crítica, 1988.

2 Daniel Kalpokas, "La incognoscibilidad y los límites del sentido", en: AA.VV., Peirce, Schiller, Dewey y Rorty. Usos y revisiones del pragmatismo clásico, Buenos Aires, Dcl signo, 2009, pp. 105-125. 
procuraré mostrar que los argumentos esgrimidos por Kalpokas se asientan, por un lado, sobre una interpretación inadecuada de los textos de Peirce y, por el otro, sobre una comprensión deficitaria del uso pragmático universal de la teoría de los actos de habla (4).

1.

Peirce distingue dos posibles definiciones del término "realidad". Una definición oscura o abstracta y una definición pragmática o basada en la crítica del sentido de los signos lingüísticos. La primera definición se encuentra formulada en la siguiente afirmación. "Podemos definir lo real como aquello cuyas características son independientes de lo que cualquiera puede pensar que son" (C.P.: 5. 405). Esta definición de "lo real" puede originarse en una cuasi-reflexión sobre la diferencia entre las nociones de realidad y de ficción. Una ficción depende absolutamente del pensamiento de su autor. Éste determina arbitrariamente las propiedades que la definen. Por el contrario, algo real debería pensarse como algo totalmente independiente de lo que pueda pensar cualquier sujeto empírico (tú o yo). Según Peirce esta primera definición contiene aún una idea oscura sobre la realidad. Su oscuridad radica en que puede conducirnos a pensar que la realidad es totalmente independiente del pensamiento humano en general, en el sentido de que algunas propiedades de lo real serían por principio incognoscible para el pensamiento humano. Esta conclusión carece de sentido porque quien la sostiene pretende, por un lado, que lo real puede ser incognoscible y, por el otro, que él tiene permitido formular una definición verdadera de la realidad. Tal conclusión autocontradictoria se puede evitar mediante la respuesta a la siguiente pregunta: ¿qué significa decir que lo real es independiente de toda opinión? Según Peirce, ello no puede significar "independiente del pensamiento humano en general", sino sólo "independiente de lo que tú, yo o cualquier número finito de seres humanos pensamos".

Si la mencionada definición abstracta de "realidad" es efectivamente una idea oscura y nosotros queremos aclararla, podemos atender al método propuesto por Peirce para aclarar ideas, o sea, aplicar la máxima pragmática que él formuló del siguiente modo: "Consideremos qué efectos que puedan tener concebiblemente repercusiones prácticas concebimos que tiene el 
objeto de nuestra concepción. Nuestra concepción de estos efectos es pues el todo de nuestra concepción del objeto" (C.P.: 5. 402)33. Según Peirce, quien quiera aclarar sus ideas sobre una cosa debe preguntarse por los probables efectos perceptibles de la misma. Ninguna propiedad de una cosa real, sobre la cual podamos qucrer alcanzar una idea clara, puede carecer de efectos perceptibles. Ello significa que carece absolutamente de sentido hablar de propiedades o de cosas que fuesen para nosotros por principio incognoscibles. Peirce ilustra la aplicación de su máxima pragmática mediante algunos ejemplos. Se pregunta: ¿qué queremos decir nosotros cuando calificamos de "dura" o de "pesada" a una cosa, o cuando hablamos de una "fuerza"? Mediante la aplicación de la máxima pragmática, Peirce muestra que una clara definición de estos términos no puede referirse a presuntas propiedades carentes de efectos perceptibles. En rigor, los efectos de una cosa agotan sus propiedades y carecería de sentido hablar sobre algo que no tenga efectos o de algo cuyos efectos fuesen para nosotros incognoscibles por principio.

La máxima pragmática puede ser útil para aclarar el significado del término "realidad" y evitar la oscuridad de su mencionada definición abstracta. Si aplicamos la máxima pragmática a la idea de realidad, la pregunta decisiva sería: ¿Cuáles son los efectos de las cosas reales en general? Peirce responde a esta pregunta del siguiente modo:

Apliquemos pues aquí nuestras reglas. Según éstas, la realidad como cualquier otra cualidad, consiste en los efectos sensibles específicos que producen las cosas que participan de la misma. El único efecto que tiene las cosas reales es el de causar creencia, pues todas las sensaciones emergen a la conciencia en forma de creencias (C.P.: 5. 406).

Mediante la aplicación de la máxima pragmática Peirce muestra que lo real consiste en los efectos perceptibles que se producen en la conciencia

${ }^{3}$ Sobre la formulación de esta máxima véase: W. H. F. Barnes, "Peirce on How to Make Our Ideas Clear", en: Wiener Ph. P./Young F. H. (Eds.), Studies in the Philosophy of Charles Sanders Peirce, Cambridge, Massachusets, 1952; Karl-Otto Apel, Der Denkweg von Charles Sanders Peirce. Eine Einfiubrung in der amerikanischen Pragmatismus, Frankfurt a. M., 1975, pp. 133-146. 
humana. Todas las propiedades de las cosas reales deben poder producir una creencia en el sujeto de conocimiento. Ello significa que carecería de sentido hablar sobre una propiedad de una cosa real que no pudiese producir ningún efecto en nuestra conciencia. Naturalmente, esto no significa que todas nuestras creencias se correspondan con algo real. Solamente las "verdaderas creencias" o las "creencias en lo real" corresponden a las cosas reales. Las creencias falsas, por el contrario, no se corresponden a lo real, porque ellas son sólo "creencias en una ficción". En este punto de su argumentación, Peirce conecta la noción de realidad con la noción de verdad, la que también puede aparecer oscuramente en la conciencia de los seres humanos y puede ser aclarada mediante la máxima pragmática.

Según Peirce, la diferencia entre "creencia en lo real" y "creencia en una ficción" remite a distintos métodos de fijación de creencias. En su artículo "La fijación de la creencia" (1877), Peirce distingue cuatro métodos utilizados por los seres humanos para fijar creencias: el método de la tenacidad, el método de la autoridad, el método metafísico (que Peirce denomina "a prior") y el método lógico o científico ${ }^{4}$. En el marco de la presente exposición dejaré a un lado los primeros dos métodos, para detenerme brevemente sobre la diferencia entre los dos últimos.

Quien utiliza el método metafísico de fijación de creencias, presupone que puede legítimamente decidir qué afirmaciones son verdaderas y qué enunciados son falsos. De acuerdo a este presupuesto, cualquier individuo estaría habilitado para seleccionar sus creencias libremente, movido por sus preferencias personales. Por cierto que para desarrollar y educar sus preferencias naturales los individuos pueden dialogar entre sí y cada uno puede dejarse influenciar por las opiniones ajenas. De esta manera, cada individuo podría considerar cuidadosamente cada problema sobre el que reflexione, desde diversas perspectivas, antes de decidirse a fijar una creencia como su personal solución al problema. En esta reflexión individual, el

${ }^{4}$ Véase: J. Krawitter, Charles Sanders Peirce. Realität, Wabrheit, Gott, Würzburg, 1984, pp. 105 ss.; David Wiggins, "Refexions on Inquiry and Truth Arising from Peirce' s Method for Fixation of Belief", en: Cheryl Misak (ed.), The Cambridge Companion of Peirce, Cambridge, Cambridge University Press, 2004, pp. 87-126. 
único criterio orientador aplicable para decidir entre dos afirmaciones contradictorias sería lo que cada uno cree más apropiado para su propio entendimiento. Por ello, el resultado de la aplicación del método metafísico de fijación de creencias es que cada uno cree en lo que cada uno encuentra más adecuado a su entendimiento. El problema de la elección entre dos afirmaciones contradictorias parece reducirse, mediante este método, a una cuestión de gusto subjetivo; en una palabra, a un asunto privado de cada cual. Cada individuo podría, entonces, apelar a su conciencia subjetiva para elegir entre dos afirmaciones contradictorias.

Seguramente, los individuos pueden hablar entre sí para ayudarse a formar una opinión sobre algún asunto, pero, en el marco del método metafísico de fijación de la creencia, el diálogo argumentativo no es considerado como el único camino seguro para encontrar la única solución correcta de un problema. Peirce caracteriza del siguiente modo a quienes usan el método metafísico. "No parece que estas mentes crean que la disputa tenga que cesar alguna vez; parecen pensar que la opinión que es natural para uno no lo sea así para otro, y que, en consecuencia, nunca se establezca creencia" (C.P.: 5. 406).

Según Peirce, el método metafísico tiene muchas ventajas frente a los métodos de la tenacidad y de la autoridad, por lo que afirma que "en la medida en que no pueda aplicarse ningún método mejor, debe seguirse éste" (C.P.: 5.383). Sin embargo, el método metafísico tiene el siguiente defecto decisivo: mediante la aplicación del método metafísico no pueden distinguirse las creencias verdaderas de las creencias falsas, o sea, las creencias en lo real de las creencias en una ficción. Esta distinción sólo puede establecerse mediante la aplicación del cuarto método de fijación de creencias, denominado por Peirce "método lógico o científico". A diferencia del método metafísico, el método científico supone, por un lado, que las creencias no deben ser determinadas por lo que le resulte aceptable a la conciencia de cada individuo, sino por "algo permanente externo" a la conciencia de cada cual, algo independiente de lo que tú, yo o cualquier número finito de seres humanos piensen ${ }^{5}$.

${ }^{5}$ Véase: F. E. Reilly s.j., Charles Peirce's Thoeory of Scientific Method, New York, 1970; Stefan Kappner, "Why Should We Adopt The Scientific Method? A Response to 
Cuando los investigadores científicos buscan la solución de un problema referente a algo real, suponen necesariamente que la solución a dicho problema no es un asunto privado, que cada uno debe resolver como le parece. Seguramente, cuando distintos investigadores comienzan a ocuparse de un problema empírico, cada uno puede tener su propia opinión sobre el asunto, o sea su propia propuesta de solución del problema. Ello significa que en el punto de partida de una investigación empírica, cada investigador puede tener su hipótesis preferida, testeada mediante sus experimentos preferidos. Pero, a diferencia de lo que ocurre con el método metafísico, se espera que el método científico conduzca a todos los investigadores a una única conclusión, a saber: la única respuesta correcta al problema investigado. Esa conclusión común, que en toda investigación empírica debe ser presupuesta por todo posible investigador como el objetivo último del proceso de investigación, el lo que Peirce denomina opinión "última, final o predestinada" (C. P.: 5.383).

Según Peirce, el objetivo de un proceso de investigación empírica no puede ser sólo la refutación de un siempre creciente número de hipótesis, sino que también debe necesariamente suponerse una progresiva convergencia entre las opiniones de todos los posibles investigadores que se ocupan del mismo problema referente a lo real. La noción peirceana de "opinión última" implica, entonces, lo siguiente. Las opiniones de los distintos seres humanos que se ocupan de un mismo problema referente a algo real y aplican el cuarto método (lógico o científico) de fijación de las creencias deben, en el largo plazo, converger en una única opinión última. El resultado de un proceso colectivo de investigación es una convergencia aproximativa entre las opiniones sobre lo real. El punto ideal hacia el que todas las opiniones deben necesariamente converger es una opinión última, o sea una opinión ya incuestionable y por tanto verdadera para quienes participamos del proceso de investigación. "La opinión destinada a que todos los que investigan estén por último de acuerdo en ella es lo que

Misak's Interpretation of Peirce's Concept of Belief', Transactions of the Charles S. Peirce Society, 2000, XXX, 2, pp. 253-270. 
significamos por verdad, y el objeto representado en esta opinión es lo real" (C. P.: 5. 408) ${ }^{6}$.

De esta manera, Peirce conecta su definición pragmática del término "realidad" con su noción de "opinión última": lo real es el objeto de una opinión última, que debe ser creída por todo posible investigador en el largo plazo, o sea que debe ser reconocida unánimemente como verdadera. La opinión última sobre una cosa real es para nosotros una opinión verdadera que implica un consenso último sobre lo real entre todos los posibles miembros de una comunidad de investigación.

Cuando Peirce sostiene que la opinión última está "destinada" a ser aceptada o la llama "opinión predestinada", estas expresiones no deben entenderse en el sentido de una predicción de que todo proceso de investigación alcanzará efectivamente una opinión última. Estas expresiones significan sólo que los miembros de una comunidad de investigación no pueden orientar arbitrariamente su investigación, según sus preferencias individuales. Por ello se dice que la actividad del pensamiento colectivo sobre lo real mediante la aplicación del método científico de fijar creencias puede aparecer a la conciencia de los individuos como la fuerza del destino.

2.

A mi parecer, pueden distinguirse dos aspectos en la noción peirceana de "opinión última". Por un lado, Peirce utiliza esta idea dentro de un argumento reflexivo, que pretende examinar críticamente el sentido de los signos lingüísticos. Este argumento contiene la mencionada aplicación de la máxima pragmática para aclarar las ideas de "realidad" y de "verdad". Este argumento debería expresarse en modo condicional, diciendo por ejemplo: "una afirmación verdadera es aquella que, como una opinión última de un

' Véase: Peter Skagestad, The Road of Inquiry. Charles Peirce's Pragmatic Realism, New York, Columbia University Press, 1981, pp. 62-72; Karl-Otto Apel, "Pragmatismus als sinnkritischer Realismus auf Basis regulativer Ideen. In Verteidigung einer Peirceschen Theorie der Realität und Wahrheit", en: M. L Raters / M. Willaschek (eds.), Hilary Putnam und die Tradition des Pragmatismus, Frankfurt a. M., Suhrkamp, 2002, pp. 117-147. 
proceso de investigación, debería ser admitida, en el largo plazo, como incuestionable"; o diciendo también: "una cosa real sería el objeto de una opinión última". Este modo de expresión condicional es quizás suficiente para explicitar el significado de los términos "realidad" y "verdad" mediante un examen crítico del sentido de los signos lingüísticos, o, en términos de Peirce, mediante la aplicación de la máxima pragmática para aclarar nuestras ideas.

Además del aspecto mencionado, que podría denominarse "críticosemiótico", el argumento de Peirce contiene otro aspecto, el "epistemológico", que puede presentarse mediante la siguiente tesis: nosotros, los posibles participantes de una comunidad de comunicación tenemos efectivamente la capacidad de conocer lo real; o, dicho de otro modo, lo real es efectivamente cognoscible para nosotros. Yo quisiera destacar este segundo aspecto del argumento de Peirce, donde aparece la noción de opinión última. Este argumento no trata sólo de lo que los investigadores conocerían en el largo plazo; no es sólo una suerte de experimento mental sobre una situación por principio inalcanzable. El argumento de Peirce se refiere también a las capacidades cognitivas, que realmente tienen los investigadores para poder conocer lo real y las capacidades que no tienen ${ }^{7}$. Este segundo aspecto del argumento de Peirce no presupone, ciertamente, que nosotros, los posibles miembros de la comunidad de investigación, alcanzaremos efectivamente en el futuro la opinión última sobre algún determinado problema empírico. Sin embargo, mediante este argumento Peirce pretende fundamentar que la opinión última sobre una cosa real (o sea la respuesta verdadera a un problema empírico) es para nosotros necesariamente alcanzable, aunque uno a priori no pueda saber si nosotros, los posibles miembros de una comunidad de investigación, lo alcanzaremos efectivamente.

A mi juicio, hay tres aspectos de esta concepción que vale tener en cuenta. En primer lugar, quisiera resaltar que, según Peirce, en el marco del método metafísico de fijación de creencias, el calificativo "verdadero" sólo puede tener un valor enfático. El método científico, por el contrario, permite distinguir las creencias verdaderas (creencias en lo real) de las

${ }^{7}$ Cfr. Charles Sanders Peirce, "Some consequences of four incapacities" (1868). 
creencias falsas (creencias en una ficción), o sea permite identificar el camino correcto para alcanzar una opinión última sobre lo real.

En segundo lugar, quisiera destacar que la definición abstracta de lo real como "independiente de nuestro pensamiento" puede conducirnos a la paradójica representación de algo totalmente independiente del pensamiento humano en general, o sea, a la representación de algo absolutamente incognoscible para nosotros. Para evitar la paradójica situación de pretender formular una afirmación verdadera sobre algo que presuntamente no podríamos conocer y acerca de lo cual, por tanto, tampoco podríamos formular afirmaciones verdaderas, Peirce nos ofrece una definición pragmática de lo real. De acuerdo con esta definición lo real sería el objeto de una opinión última que es siempre ya alcanzable para nosotros en el largo plazo. La distinción conceptual entre ficción y realidad puede trazarse sin paradojas mediante la diferencia entre "lo que depende de lo que tú, yo y cualquier número finito de seres humanos pensemos", por un lado, y "lo que depende del pensamiento humano en general (o sea el objeto de una opinión última"), por el otro. De esa manera, puede pensarse lo real como algo independiente de $m i$ pensamiento sin suponer que exista algo real que sea absolutamente incognoscible ${ }^{8}$.

Por último, quisiera señalar que la idea de opinión última, que Peirce concibe en el marco de la lógica de la investigación científico natural, puede ser pensada como un presupuesto más general, necesariamente admitido como objetivo de todos los participantes de todo posible diálogo argumentativo, independiente del tema que trate. En este mismo momento, por ejemplo, nosotros (el lector y yo) estamos tratando de examinar un problema filosófico. Nos interesa determinar la validez de los argumentos con los que pretendieron fundamentar sus tesis y para ello, participamos de

${ }^{8}$ En rigor podría hablarse de una dependencia recíproca del pensamiento humano con la realidad. Sobre esta dependencia véase: Daniel Kalpokas, "El problema del conocimiento del mundo externo de Kant a Peirce", en: G. Fernández / D. Parente (Eds.), El legado de Immanuel Kant. Actualidad y perspectivas, Mar del Plata, Suarez, 2004, pp. 83-89; Alberto Mario Damiani, "Der Pragmatismusstreit und der sinnkritische Realismus", en: id., Handlungswissen. Eine transzendentale Erkundung nach der sprachpragmatischen Wende, Freiburg/München, Verlag Karl Alber, 2009, pp. 230-242. 
un diálogo argumentativo. Nosotros participamos de este diálogo, pero este "nosotros" no se limita a quien escribe y lee estas líneas ahora (yo y tú) sino que se extiende, por ejemplo a los pragmatistas que publicaron los argumentos que estamos examinando, también se extiende a los autores examinados por los pragmatistas y en rigor a todos los que alguna vez se interesaron por los problemas filosóficos que hoy discutimos una vez más.

Pero la extensión de este "nosotros" no se detiene tampoco allí, sino que abarca a todos los que pueden y también a todos los que podrían participar de este diálogo argumentativo sobre las respuestas pragmatistas a ciertos problemas filosóficos. Pues bien, todos los posibles participantes de este diálogo argumentativo son miembros de una comunidad de comunicación que tiene un objetivo muy preciso, que es encontrar la solución de los problemas discutidos. Esa solución es, precisamente, la opinión última que buscamos en este momento. No podemos saber a priori si la comunidad de argumentación de la que participamos mediante nuestros argumentos actuales alcanzará efectivamente en el futuro el fin que actualmente buscamos y, sin embargo, dedicamos tiempo, entusiasmo y trabajo para que la comunidad de argumentación seleccione los mejores argumentos y descarte los peores. ¿Tiene sentido lo que estamos haciendo en este preciso momento, cuando nos esforzamos en un trabajo colectivo sin ninguna garantía de que alguno de los aquí presentes llegue en vida a conocer la solución de los problemas que examinamos? Esta pregunta es difícil de responder, pero creo que Peirce nos animaría a seguir trabajando, diciéndonos que podemos estar seguros de que la comunidad de comunicación a la que siempre ya pertenecemos puede encontrar la respuesta verdadera a los problemas que estudiamos, puede alcanzar, en definitiva, la opinión última e incontrovertible que soluciona estos problemas.

3.

Diez años antes de publicar los textos examinados en el apartado anterior, Peirce había esbozado algunas ideas sobre el problema de la cognoscibilidad de lo real, entendido como una pregunta por la existencia de una facultad humana capaz de conocer la realidad. Nos referimos a una página dedicada a la cuestión sexta de su trabajo "Questions concerning 
certain faculties claimed for man" (1868). Se trata de un texto temprano, perteneciente a un período anterior a la formulación de la máxima pragmática y a los otros elementos que le permitirán elaborar la definición de la noción de realidad mediante una crítica del sentido de los signos, tal como aparece en los trabajos examinados en el apartado anterior. Alguna de las afirmaciones contenidas en esa página pueden resultar útiles para confirmar lo dicho hasta aquí.

En primer lugar es importante detenerse en el enunciado mismo de la cuestión sexta. Peirce la presenta del siguiente modo: "Establecer si un signo puede tener algún sentido cuando por su definición es el signo de algo absolutamente incognoscible". Esta formulación indica claramente que esta cuestión trata sobre el mismo problema que Peirce examinará diez años después, en el último apartado de "How to make our ideas clear". En ambos casos se trata de saber si tiene sentido hablar de "algo absolutamente incognoscible". La tesis que Peirce defiende en ambos casos es que algo absolutamente incognoscible, no puede concebirse con sentido. Puede decirse que el argumento presentado en el breve texto de 1868 carece de la elaboración y la claridad que encontramos en el texto de 1878, examinado más arriba. En la sexta cuestión de 1868 no aparece la máxima pragmática, ni la comunicad de investigadores e intérpretes de signos, ni el principio de convergencia, ni la idea de una opinión última de la investigación. En la cuestión sexta Peirce se limita a señalar que "obtenemos todas nuestras concepciones por medio de abstracciones y combinaciones de cogniciones, que se dan en primer lugar en juicios de experiencia". Si esto fuese todo lo que Peirce hubiese dicho en su obra para probar la cognoscibilidad de lo real, no podríamos calificar su concepción de pragmatista o "pragmaticista", como terminó denominándola para diferenciarse de algunos de sus admiradores que la habrían interpretado incorrectamente. Por ello, si se quiere reconstruir adecuadamente los argumentos de Peirce sobre la cuestión de lo incognoscible no resulta aconsejable limitarse a la breve explicación de la cuestión sexta de 1868.

Es más: si se quisiera presentar una reconstrucción más o menos completa de las ideas de Peirce sobre la cuestión de la cognoscibilidad de lo real en el año 1868, es necesario atender a otro texto de esa mismo año: "Some consequences of four incapacities". Allí sostiene Peirce lo siguiente: 
Si el significado de una palabra reside en el concepto que ćsta transmite, lo absolutamente incognoscible no tendrá entonces ningún significado, puesto que no se corresponde con ningún concepto. Se trata, por tanto, de una expresión carente de significado; y, consiguientemente, signifique lo que signifique un término como 'lo real', será siempre cognoscible en un cierto grado y pertenecerá así a la naturaleza del conocimiento en el sentido objetivo del término (C. P.: 5. 257).

Este pasaje muestra claramente que ya desde 1868, Peirce no se pregunta si el término "incognoscible" tiene sentido. Resulta obvio que le atribuye algún sentido a ese término, en la medida en que lo utiliza. Tal como muestra el pasaje citado, el interés de Peirce se centra en el sentido de la expresión "lo real". Su tesis es que la cognoscibilidad se encuentra necesariamente entre las notas que permiten definir correctamente esa expresión. Por lo tanto, la cuestión de la cognoscibilidad de lo real, y no el sentido del término "incognoscible" es lo que preocupa a Peirce en estos textos de 1868, tal como atestigua tanto el pasaje citado como el enunciado de la cuestión sexta, mencionado más arriba.

En la breve explicación que sigue a la formulación de la cuestión sexta en "Questions concerning certain faculties claimed for man" hay un pasaje en el que Peirce sostiene que el concepto de "algo absolutamente incognoscible" es contradictorio: "En consecuencia, no cognoscible, si se trata de un concepto, es un concepto de la forma 'A, no A', y es, por lo menos, contradictorio en sí mismo". Si se atiende a la formulación de la cuestión sexta, se advierte que Peirce sostiene en este pasaje que el concepto de una realidad incognoscible entraña algún tipo de contradicción. Las razones que justifican esta tesis son en parte formuladas por Peirce en sus escritos posteriores, entre ellos los que examinamos en los apartados anteriores. Una combinación de una reconstrucción bienintencionada de los argumentos que contienen esas razones, por un lado, y del uso correcto de instrumentos de análisis filosófico que surgen en la historia de la filosofía con posterioridad a la muerte de Peirce, por el otro, permite explicar en qué sentido puede decirse que la afirmación de una "realidad incognoscible" contiene algo contradictorio. 
4.

En su reciente trabajo "Lo incognoscible y los límites del sentido", Daniel Kalpokas sostiene que Peirce se equivoca al pretender alcanzar, mediante una crítica del sentido, la conclusión de que la realidad es por principio cognoscible. La estrategia elegida por Kalpokas para criticar la tesis de la cognoscibilidad de lo real adolece, a mi juicio, de algunas dificultades que quisiera señalar brevemente a continuación. La primera dificultad es de índole hermenéutica y consiste en que, por un lado, centra su análisis, casi exclusivamente, en el breve texto que sigue a la sexta cuestión de "Questions Concerning Certain Faculties Claimed for Man", sin atender ni a la formulación de esa misma cuestión, ni al artículo del mismo año ya mencionado y, por el otro, intercala de manera anacrónica afirmaciones provenientes de los textos de 1877 y 1878. Este recorte injustificable de la base textual le permite a Kalpokas atribuirle a Peirce una ambigüedad que ningún lector bienintencionado podría encontrar.

Como acabamos de indicar, en la formulación de la cuestión sexta, Peirce se pregunta por el sentido de un signo de algo absolutamente incognoscible y en el otro artículo del mismo año afirma que el referente de la expresión “lo real” es cognoscible. En vez de tener en cuenta seriamente estos pasajes, donde Peirce explicita que su interés se centra en la cognoscibilidad de lo real, a Kalpokas le resulta más sencillo apresurarse a diagnosticar que "la ambigüedad del blanco de ataque parece ser una constante en los textos peirceanos". La ambigüedad que Kalpokas cree encontrar en estos textos consiste en lo siguiente. Según Kalpokas, no se sabe si Peirce afirma que el término "incognoscible" carece de significado, o si afirma que "aquello que se postula como incognoscible" carece de sentido. En realidad lo que Kalpokas denomina "primera posibilidad hermenéutica", esto es, que el término "incognoscible" carece de significado no es una tesis que Peirce haya defendido. Kalpokas, sin embargo, no se contenta con atribuirle esta tesis a Peirce, sino que se entretiene en refutarla en varios párrafos de su trabajo. En su presunta refutación de Peirce, Kalpokas le presenta al lector como argumentos las siguientes obviedades:

9Daniel Kalpokas, "La incognoscibilidad y los límites del sentido”, cit., p. 111. 
Lo cierto es que "incognoscible" no es un ruido sin sentido para nosotros. Prueba de ello es que puede ser utilizado significativamente en distintas aserciones (por ejemplo al decir "No hay incognoscibles"). De hecho el propio Peirce hace uso del término "incognoscible" para formular el problema que pretende tratar, a saber, si un signo puede aludir a lo que es absolutamente incognoscible ${ }^{10}$.

A pesar de la dificultad hermenéutica señalada, el mencionado artículo de Kalpokas tiene el mérito de intentar determinar en qué consiste la "contradicción" que Peirce descubre en la expresión "realidad incognoscible". Kalpokas acierta también al orientar inicialmente ese intento comprendiendo el término "contradicción" más allá de su acepción sintáctico-semántica y remitir a la noción de contradicción pragmática o performativa, tal como aparece en las obras de Karl-Otto Apel y Jürgen Habermas. Kalpokas introduce esta noción en el siguiente pasaje:

Considérese el siguiente enunciado: (1) 'Hay incognoscibles.' Si el contenido proposicional de (1) se usa para realizar una pretensión de saber, entonces, al explicitar el contenido performativo de este enunciado, tendríamos la siguiente aserción: (2) '[Sé que] hay incognoscibles. ${ }^{11}$

Una vez que se explicita el uso que se pretende hacer del contenido proposicional especificado en 1), se pone de manifiesto el carácter autocontradictorio de 2). Aquí aflora de un modo claro la contradicción que está involucrada en el uso del término "incognoscible". Una manera de entender dicha contradicción es interpretarla -a la luz de las obras de Apel y Habermas- como una contradicción pragmática. El análisis de los actos de habla realizado por estos autores pretende exhumar las implicaciones que, para el significado y la validez de lo que decimos, posee la dimensión pragmática del lenguaje. Para estos autores, una contradicción pragmática (o performativa) es una contradicción que se produce entre lo que Austin llamaba el "contenido locutivo" y el "contenido ilocutivo" del acto de habla.

${ }^{10}$ Ibid., pp. 112-113.

11 "Entre corchetes se expresa el contenido performativo de las aserciones" (esta nota pertenece al texto de Kalpokas citado. A. M. D.). 
Se trata de una contradicción que tiene lugar entre el contenido semántico de lo que decimos, y lo que hacemos en el mismo acto de decirlo ${ }^{12}$.

En este pasaje aparece de manera patente la segunda dificultad del trabajo de Kalpokas. La misma consiste en el grave error de confundir el verbo "saber" con un verbo performativo.

Según la teoría de los actos de habla, hay ciertos verbos que utilizados en primera persona del singular del presente del indicativo, permiten explicitar la acción lingüística que realizamos al decir algo ${ }^{13}$. En términos de esta teoría estos verbos expresan la "fuerza ilocucionaria" de los actos de habla o permiten realizar "actos ilocucionarios". Por ejemplo, los actos de prometer, advertir, ordenar, afirmar, preguntar, confesar, etc. algún contenido proposicional cualquiera (p), pueden realizarse al decir: "Te prometo que $\mathrm{p}$ ", "Les advierto que $\mathrm{p}$ ", "Le ordeno que p", "Afirmo que $\mathrm{p}$ ", "Pregunto si p", "Te confieso que p", etc. Es cierto que estas acciones pueden realizarse en ciertos contextos de enunciación sin necesidad de utilizar esos verbos. En muchos casos una cierta combinación de factores extralingüísticos le indica al oyente el tipo de acto realizado por un hablante. Por ello, no es siempre necesario usar esos verbos para dar a entender la fuerza ilocucionaria de nuestros actos de habla. Esos factores pueden ser, según los casos, la entonación, el contexto, la relación entre el hablante y el oyente, las expectativas mutuas de los interlocutores, etc. Sin embargo, también hay casos en los que al oyente no le resulta del todo clara la fuerza ilocucionaria de una expresión, casos en los que se produce lo que podría denominarse una "ambigüedad performativa". Si en esos casos el oyente le hace saber al hablante que su expresión es performativamente ambigua, éste puede explicitar la fuerza ilocucionaria de su acto de habla mediante un verbo performativo, en primera persona del presente del indicativo. De esta manera indica claramente el tipo de acto que realiza al decir p. Esta explicitación se lleva a cabo, entonces, cuando el hablante dice, por ejemplo: "Te prometo que $\mathrm{p}$ ", "Les advierto que $\mathrm{p}$ ", "Le ordeno que $\mathrm{p}$ ", “Afirmo que p",

12 Op. cit., pp. 118-119.

13 Véase: Austin, J. L.: How to Do Things with Words, Oxford, 1962; Searle, John: Speech Acts, Cambridge, 1969. 
"Pregunto si p", "Te confieso que p", etc. Adviértase que en todos estos casos el hablante realiza ciertos actos al usar ciertos verbos, esto es, al decir "prometo", promete, al decir "advierto" advierte, etc. Por ello, estos verbos se denominan performativos o realizativos. De más está decir que no todos los verbos permiten realizar acciones lingüisticas.

Karl-Otto Apel y Jürgen Habermas han utilizado algunos descubrimientos de la teoría de los actos de habla formulada por John L. Austin y John R. Searle para formular, respectivamente, sus propuestas de una pragmática trascendental y una pragmática universal ${ }^{14}$. No es éste el lugar para reconstruir y valorar ese uso. Baste aquí señalar que el mismo permite advertir que la parte performativa de los actos de habla no sólo contiene la fuerza ilocucionaria de los verbos realizativos sino un conjunto de presupuestos necesarios, admitidos implícitamente por el hablante y el oyente. La necesidad de los mismos puede comprobarse del siguiente modo. $\mathrm{Si}$ se intenta negar estos presupuestos se produce un tipo de inconsistencia denominada "autocontradicción pragmática" o "autocontradicción performativa". La misma consiste en una contradicción entre las dos partes de un acto de habla, esto es, entre la parte performativa y la parte proposicional ${ }^{15}$.

14 Véase, por ejemplo: Apel, Karl-Otto: Transformation der Philosopbie, 2 Bde. Frankfurt a.M., 1973; id., "Das Problem der philosophischen Letzbegündung im Lichte einer tranzendentalen Sprachpragmatik. Versuch einer Metakritik des 'Kritischen Rationalismus'.' in: Kanitscheider, B. (Hrsg.), Sprache und Erkenntnis; Innsbruck, 1976, S. 55-82; id., "Illokutionäre Bedeutung und normative Gültigkeit. Die transzendentalpragmatische Begründung der uneingeschränkten kommunikativen Verständigung", in: Gerhard Preyer, et al. (Hrsg.), Intention Bedeutung - Kommunikation. Kognitive und bandlungstheoretische Grundlagen der Sprachtheorie, Opladen, 1997, S. 288-303; id. Auseinandersetzungen in Erprobung des transzendentalpragmatischen Ansatzes, Frankfurt a.M., 1998; Jürgen Habermas, "Was heißt Universalpragmatik", in: K. O. Apel (Hrsg.) Sprachpragmatik und Pbilosopbie, Frankfurt a.M., 1976, S.174-272; id., Theorie des kommunikativen Handelns, Frankfurt a. M., 1981, Band 1.

15 Véase: Wolfgang Kuhlmann, Reflexive Letzbegrünndung, Untersuchungen zur Transzendentalpragmatik, Freiburg/München, Karl Alber Verlag, 1985; Matthias M. Kettner, "Ansatz zu einer Taxonomie performativer Selbswidersprüche", in: Dorschel A. et al. (Hrsg.) Transzendentalpragmatik. Ein Symposion für Karl Otto Apel, 
En el pasaje antes citado, Kalpokas afirma que la expresiôn "[sé que] hay incognoscibles" es pragmáticamente autocontradictoria. Esta afirmación se basa en suponer, tal como señala explícitamente en la nota al pie de página, que con la expresión "[Sé que]" se ha explicitado la parte performativa de un acto de habla, cuya parte proposicional es "hay incognoscibles". Sin embargo, este supuesto es falso porque el verbo "saber" no indica lo que el hablante hace al decir "bay incognoscibles". En términos de la mencionada teoría de los actos de habla, el verbo "saber" no es un verbo realizativo o performativo. Como señalé más arriba, los verbos performativos, tales como "prometer", "advertir", "ordenar", "afirmar", "preguntar", "confesar", etc. indican el tipo de acto que realiza el hablante al utilizarlos en primera persona del singular del presente del indicativo. Para comprender la diferencia entre el verbo "saber" y los verbos performativos, basta advertir que quien dice "prometo tal o cual cosa" está prometiendo, pero nadie sabe algo por el solo hecho de decir "sé tal o cual cosa". Para prometer algo basta con decir "prometo", pero para saber algo no basta con decir "sé".

La expresión "sé que hay incognoscibles", al igual que "hay incognoscibles", presenta sólo el contenido proposicional de un acto de habla, cuya fuerza ilocucionaria permanece aún implícita y podría explicitarse mediante un verbo performativo, por ejemplo "afirmo que" o "prometo que" o " confieso que" o "apuesto a que" o "me pregunto si", etc. ${ }^{16} \mathrm{Si}$ se explicitase la parte performativa de la expresión "sé que hay incognoscibles", podrían determinarse tanto la fuerza ilocucionaria del acto de decirla como los presupuestos pragmáticos implícitos y sus condiciones necesarias de sentido. Recién cuando estos presupuestos sean explicitados podría evaluarse si hay o no hay alguna inconsistencia entre las dos partes

Frankfurt a.M., Suhrkamp, 1993, pp. 187-211.

${ }_{16}$ Por una parte, puede suponerse que la acción del hablante, en este caso, consiste en afirmar ( $y$ no prometer, confesar, apostar, etc.). Por otra parte, debe suponerse que un hablante competente sabe el tipo de acción que realiza. El contenido de ese saber no es la parte proposicional de su acto de habla, sino la parte performativa. Por ello, puede denominarse "saber performativo" al saber que le atribuimos al hablante sobre sus propios actos de habla y las condiciones de sentido de los mismos. Sobre este saber, véase: Alberto Mario Damiani, Handlungswissen. Eine transzendentale Erkundung nach der sprachpragmatischen Wende, cit., especialmente pp. 60-104. 
del acto de habla, entre el contenido proposicional y el acto de afirmarlo, esto es, si el mismo contiene una autocontradicción performativa o no.

La confusión del verbo "saber" con un verbo performativo no sería tan grave si las críticas de Kalpokas al argumento de Peirce no se asentasen sobre ella. Partiendo de esta confusión Kalpokas propone distinguir entre la pretensión de verdad y lo que denomina "pretensión de conocimiento". Como es sabido, tanto la pragmática universal de Habermas como la pragmática trascendental de Apel combinan el mencionado uso de la teoría de los actos de habla con una arquitectónica de las pretensiones de sentido y validez que se encuentran implícitas en la dimensión performativa de los actos de habla. La pretensión de verdad es la pretensión de validez que adquiere primacía en los actos de habla asertóricos. En ellos el hablante afirma como verdad un contenido proposicional, esto es, eleva una pretensión de verdad ante una comunidad (ideal e ilimitada) de interlocutores. Esta comunidad debe poder resolver, mediante argumentos, si dicha pretensión está justificada o no lo está. La perspectiva pragmático trascendental entiende el sentido de la noción de verdad no sólo en su dimensión semántica, esto es, en la relación entre el significado de los signos y su referente, sino también y sobre todo en su dimensión pragmática, esto es, en la relación entre el significado de los signos y los usuarios. Si se atiende a esta última relación, la noción de verdad puede aclararse, en un camino iniciado por Peirce, explicando el sentido de la verdad de las proposiciones en términos de un consenso posible, esto es, por principio alcanzable in the long run por la comunidad ideal e ilimitada de argumentantes ${ }^{17}$.

La teoría consensual de la verdad, que Apel formula mediante una reelaboración del pragmaticismo de Peirce, descubre una conexión necesaria entre la verdad de las proposiciones y el posible conocimiento de esa verdad por parte de quienes las enuncian y comprenden como parte de actos de habla asertóricos. La parte performativa de estos actos contiene implícita tanto la elevación de la pretensión de verdad como el presupuesto necesario de la posible resolución de la misma mediante el discurso argumentativo de

${ }^{17}$ Cfr. Karl-Otto Apel, Auseinandersetzungen in Erprobung des transzendentalpragmatischen Ansatzes, cit., pp. 81-193. 
la comunidad ideal e ilimitada. Dentro de esta concepción pragmática, el conocimiento de la realidad no es algo que pueda poseer u obtener una conciencia aislada, al modo del solipsismo metódico de la filosofía moderna, sino el objetivo de una tarea colectiva, en la que cada participante puede sólo pretender verdad para las proposiciones propuestas en sus actos de habla asertóricos como solución a los problemas referentes a las características objetivas de la realidad.

Kalpokas parece alejarse en tres direcciones de esta concepción de la verdad, el conocimiento y la realidad que la pragmática trascendental hereda de Peirce. En primer lugar, en una dirección cartesiana que atribuye a la conciencia del hablante la facultad de determinar si sabe o no sabe algo sobre la realidad. En segundo lugar, en una dirección semanticista, que pretende que "al ámbito de la realidad es más amplio que el del conocimiento" posible. Esta dirección intenta pensar la verdad de las proposiciones con absoluta independencia de los actos de habla asertóricos, mediante los cuales los seres bumanos, como interlocutores discursivos, enuncian esas proposiciones y pretenden que son verdaderas. En términos técnicos, este intento incurre en la comisión de una falacia abstractiva. Al ignorar la parte performativa de los actos de habla asertóricos quiere concebir la verdad remitiéndola sólo a proposiciones asiladas, esto es, separadas de las acciones lingüísticas que los seres humanos realizan para conocer la realidad. Esta dirección semanticista contiene dos aspectos. Por un lado, quiere resolver el problema de la presunta incognoscibilidad de lo real mediante el siguiente bicondicional: "La afirmación 'hay incognoscibles' es verdadera si y sólo si hay incognoscibles"18. Por otro lado, deja sin aclarar la dimensión pragmática de proposiciones verdaderas sobre lo real cuya verdad fuese por principio incognoscible para la comunidad ideal e ilimitada de los investigadores, de la que somos miembros el autor y el lector de estas líneas. En otros términos, la dirección semanticista del argumento de Kalpokas deja sin responder a la siguiente pregunta pragmática: ¿Para quién serían verdaderas las proposiciones sobre la reälidad cuya verdad fuese por principio incognoscible para nosotros? En tercer lugar, Kalpokas se aleja de la concepción pragmática de la realidad en la dirección de un realismo metafisico, que postula la posibilidad de una realidad por principio inaccesible

${ }^{18}$ Daniel Kalpokas, "La incognoscibilidad y los límites del sentido", cit., p. 121. 
para nosotros. Si las nociones de verdad, conocimiento y realidad se desconectan de las acciones lingüísticas y no lingüísticas realizadas por los seres humanos que intentan conocer la realidad, reaparece el presupuesto metafísico del "ojo de Dios", que compara las proposiciones sobre la realidad (proposiciones cuya verdad se postula incognoscible para nosotros) con la realidad (incognoscible para nosotros) y corrobora la verdad de las mismas, esto es, la correspondencia entre las proposiciones y la realidad a la que se refieren ${ }^{19}$. En síntesis, Kalpokas ofrece una problemática combinación de solipsismo metódico, semanticismo y metafísica como alternativa a la definición del término "realidad", definición a la que Peirce llega mediante una crítica pragmática del sentido.

Recibido: 10/2010; aceptado: 11/2010.

19 Hilary Putnam, Reason, Truth and History, Cambridge, Cambridge University Press, 1981. 